\title{
Prebiotics as functional foods: a review
}

\begin{abstract}
Prebiotics are short chain carbohydrates that are non-digestible by digestive enzymes in humans and selectively enhance the activity of some groups of beneficial bacteria. In the intestine, prebiotics are fermented by beneficial bacteria to produce short chain fatty acids. Prebiotics also render many other health benefits in the large intestine such as reduction of cancer risk and increase calcium and magnesium absorption. Prebiotics are found in several vegetables and fruits and are considered functional food components which present significant technological advantages. Their addition improves sensory characteristics such as taste and texture, and enhances the stability of foams, emulsions and mouthfeel in a large range of food applications like dairy products and bread. This contribution reviews bioactives from food sources with prebiotic properties. Additionally, food application of bioactive prebiotics, stimulation of the viability of probiotics, health benefits, epidemiological studies, and safety concerns of prebiotics are also reviewed.
\end{abstract}

Keyword: Prebiotics; Digestibility; Fermentability; Functional food application; Health benefits 